\title{
Co-expression of Rho guanine nucleotide exchange factor 5 and Src associates with poor prognosis of patients with resected non-small cell lung cancer
}

\author{
PING HE $^{1}$, WEI WU ${ }^{1}$, HAIDONG WANG ${ }^{1}$, KELONG LIAO ${ }^{1}$, WEI ZHANG ${ }^{1}$, \\ GANG XIONG ${ }^{1}$, FENG WU ${ }^{2}$, GANG MENG ${ }^{2}$ and KANG YANG ${ }^{1}$ \\ ${ }^{1}$ Department of Cardiothoracic Surgery, Southwest Hospital, Third Military Medical University; \\ ${ }^{2}$ Institute of Pathology, Southwest Cancer Center, Southwest Hospital, Third \\ Military Medical University, 400038 Chongqing, P.R. China
}

Received August 15, 2013; Accepted September 26, 2013

DOI: 10.3892/or.2013.2797

\begin{abstract}
Specific and sensitive enough molecular biomarkers are lacking to accurately predict the survival of non-small cell lung cancer (NSCLC) patients. ARHGEF5 and Src have been shown to play an important role in tumorigenesis. However, the involvement of ARHGEF5 and Src in NSCLC remains unknown. Therefore, we evaluated the expression of ARHGEF5 and Src in resected NSCLC tissues and the correlation of co-expression of ARHGEF5 and Src and the prognosis of patients with resected NSCLC. Positive expression of ARHGEF5 was detected in 133 cases of 193 patients (68.91\%). A total of 193 NSCLC patients (male: 145; female: 48; average age: 61.84 years; age range: $31-84$ ) were enrolled in this study, of which 99 cases were squamous cell carcinomas (SCCs) $(51.30 \%)$ and 94 cases were adenocarcinomas (ADCs) (48.70\%). The expression of ARHGEF5 was mainly located in the cytoplasm of tumor cells, but not in the corresponding adjacent lung tissues. The levels of ARHGEF5 were significantly associated with age, differentiation and tumor stage. ARHGEF5 protein expression was associated with Src protein expression in NSCLC $\left(\chi^{2}=11.874, \mathrm{P}<0.01\right)$ and in ADC $\left(\chi^{2}=12.194, \mathrm{P}<0.01\right)$, but not in SCC. Co-immunoprecipitation revealed that there was a physical interaction between $\mathrm{Src}$ and ARHGEF5 in lung cancer cells. The patients with ARHGEF5(+)/Src(+) had a shorter survival time compared with the other patients (29.37 months versus 39.90 months, $\mathrm{P}=0.029)$. In conclusion, ARHGEF5/Src can be considered as a prognostic biomarker and a therapeutic target for patients with resected NSCLC.
\end{abstract}

Correspondence to: Professor Kang Yang, Department of Cardiothoracic Surgery, Southwest Hospital, Third Military Medical University, 30 Gaotanyan Street, 400038 Chongqing, P.R. China E-mail: kang_yangsw@126.com

Key words: non-small cell lung cancer, Rho guanine nucleotide exchange factor 5, co-expression, prognostic biomarker

\section{Introduction}

Lung cancer is one of the most common malignant tumors in the world, with non-small cell lung cancer (NSCLC) accounting for $80-85 \%$ of all cases. Although many methods have been developed for the treatment of NSCLC, such as surgical resection, chemotherapy and radiotherapy, the prognosis of NSCLC remains poor and the 5-year survival rate is only about $50 \%$ (1). In recent years, although many single tumor biomarkers which may be predictive of response and prognosis, including epidermal growth factor receptor (EGFR), the canonical Wnt pathway and Notch3 have been developed, there is still lack of specific and sensitive enough molecular biomarkers to accurately predict the survival of NSCLC patients (2-4).

Rho guanine nucleotide exchange factor 5 (ARHGEF5) is a member of guanine nucleotide exchange factors family (GEFs) $(5,6)$. Some studies have shown that ARHGEF5 can prompt the tumor metastasis and infiltration through its effects on adhesion and cytoskeletal functions by activating Rho GTPase $(7,8)$. Debily et al (9) revealed that ARHGEF5 is overexpressed in breast tumor, and might play a crucial role in breast tumor progression. ARHGEF5 expression leads to altered growth properties and tumorigenesis in mouse (5). Xie et al (10) also showed that ARHGEF5 mRNA levels were dramatically elevated in NSCLC cell lines compared to normal lung tissue. However, the expression of ARHGEF5 in the lung cancer tissues is unknown.

Src, a member of the Src family of tyrosine kinases, is a key regulator of cellular proliferation, survival, motility and invasiveness (11). In NSCLC, overexpression of Src has been shown to play an important role in tumor development and metastases. ARHGEF5 is crucial for Src-induced podosome formation (12). Overexpression of ARHGEF5 promotes actin stress fiber remodeling through activating RhoA, and the activation of RhoA or Cdc 42 is required for Src-induced podosome formation.

In light of the above mentioned considerations, we examined the expression of ARHGEF5 and Src in resected NSCLC tissues and evaluated the correlation between ARHGEF5 and 
Src. Furthermore, we explored the relationship between the expression of ARHGEF5 and Src and the prognosis of resected NSCLC patients.

\section{Materials and methods}

Patients and cell lines. Consecutive cohorts of NSCLC tumors resected from 193 NSCLC patients from July, 2010 to December, 2011 were analyzed. Information on baseline demographics, clinicopathological characteristics and surgical approach was collected after review of clinical notes and histopathology reports. Patients who did not receive a curative resection and had a previous history of cancer, pre-surgical chemotherapy or radiotherapy were excluded. All patients gave their written informed consent for participation in this study. This study was approved by the Southwest Hospital Ethics Committee. Another four NSCLC tissue samples as well as their corresponding normal tissues adjacent to resection margins from patients for western blot analysis were also obtained.

Normal human bronchus epithelial cell (HBE) and NSCLC cells H322, SPC-1, H1650 (adenocarcinima) and H520 (squamous cell) were obtained from Academy of Military Medical Science (Beijing, China) and maintained in RPMI-1640 or DMEM (Trace, Melbourne, Australia) supplemented with $10 \%$ fetal bovine serum (FBS), $100 \mathrm{U} / \mathrm{ml}$ penicillin, and $100 \mu \mathrm{g} / \mathrm{ml}$ streptomycin at $37^{\circ} \mathrm{C}$, in a humid atmosphere of $5 \% \mathrm{CO}_{2}$.

Tissue microarray. Tissue microarrays (TMAs) were constructed as previously described (13). Briefly, using the $\mathrm{H} \& \mathrm{E}$ sections as templates, representative areas of each tumor were identified and marked on a section of the donor block. Approximately $4 \mu \mathrm{m}$-thick (1.5-mm diameter) tissue cylinders were punched from each donor paraffin block using a TMA instrument (Beecher Instruments; Silver Spring, MD, USA). The donor cores were placed into the corresponding recipient block holes that were punched in advance using the same TMA instrument. To better represent each case, two tumor and two normal tissue cores that were located adjacent to the tumor were punched from each case. After construction, recipient blocks were placed into an oven at $37^{\circ} \mathrm{C}$ for $20 \mathrm{~min}$, rapidly removed, and pressed down by a slide for a moment to flatten the surface. Serial $4 \mu \mathrm{m}$-thick sections were cut with a Leica microtome (Leica Microsystems; Wetzlar, Germany) and mounted onto polylysine-coated slides.

Immunohistochemistry. All tissue sections were routinely dewaxed, rehydrated, and prepared for immunohistochemistry. Sodium citrate buffer ( $\mathrm{pH}$ 6.0) was used as an antigen retrieval solution, and the sections were blocked with 5\% BSA, and incubated with 1:50 polyclonal rabbit anti-ARHGEF5 (11379-1-AP, Proteintech Group), 1:50 polyclonal goat antiSrc antibody (AF3389, R\&D) overnight. The tissue sections were then incubated with secondary antibodies and DAB reagent (Gene Tech, Shanghai, China), and counterstained with hematoxylin, followed by dehydration and visualization with 3.3-diaminobenzidine (Gene Tech, Shanghai, China). Negative controls were performed in each case by replacing the primary antibody with PBS.

Expression of ARHGEF5 and Src was evaluated as the percentage of positive cells in a specimen, and by staining intensity as previously described (14). The percentage of positive cells was evaluated quantitatively and scored as 0 for staining of $\leq 1 \%$ of total cells counted, 1 for staining of $2-25 \%$, 2 for staining of $26-50 \%, 3$ for staining of $51-75 \%$, and 4 for staining of $>75 \%$ of the cells examined. Intensity was graded as follows: 0 , no signal; 1 , weak; 2 , moderate; and 3 , strong staining. A total 'staining score' of 0-12 was calculated and graded as negative (-, score $0-1)$, weak (+, score $2-4)$, moderate (++, score 5-8), or strong (+++, score 9-12). The staining score was evaluated by two experienced pathologists. Discrepant cases were reviewed in a multi-head microscope and reported as a consensus.

Fluorescent immunohistochemistry. Immunofluorescent staining was conducted to localize the expression of Src and ARHGEF5. The frozen surgically resected sections from NSCLC patients were fixed for $20 \mathrm{~min}$ in $4 \%$ paraformaldehyde. The lung tissues of patient were then rinsed 3 times with PBS for 5 min each time, and incubated in a protein-blocking solution for $20 \mathrm{~min}$ at room temperature. After incubation with the primary antibody against either human ARHGEF5 (1:50; 11379-1-AP, Proteintech Group) or human Src (1:50; AF3389, R\&D) overnight at $4^{\circ} \mathrm{C}$, followed by incubation with the secondary antibody (either myc-conjugated IgG, 1:50, Santa Cruz; or Cy3-conjugated IgG, 1:50, Beyotime) at $37^{\circ} \mathrm{C}$ for $1 \mathrm{~h}$, the cells and lung tissue of patient were counterstained with Hoechst 33342 to reveal the nuclei. Cells or slides without incubation primary antibodies were served as negative controls. The cells or slides were then analyzed by confocal laser scanning microscopy.

Western blot analysis. Tumor cells or cell lines were lysed in lysis buffer: $50 \mathrm{mmol} / \mathrm{l}$ Tris- $\mathrm{HCl}$ (pH 8.0), $150 \mathrm{mmol} / \mathrm{l} \mathrm{NaCl}$, $0.5 \%$ NP40, $0.5 \%$ sodium deoxycholate, and Protease Inhibitor Cocktail Set III (Calbiochem). The protein concentration was determined using a Bio-Rad protein assay (Bio-Rad) with bovine serum albumin as standard. Each lysate $(10 \mu \mathrm{g})$ were resolved on $10-12 \%$ denaturing polyacrylamide gels (with 3\% polyacrylamide stacking gel) and transferred electrophoretically onto a nitrocellulose membrane (GE Healthcare Biosciences). After blocking with 5\% nonfat dry milk in TBST, the membrane was incubated with a rabbit polyclonal antihuman ARHGEF5 antibody or a goat polyclonal antihuman Src antibody for $1 \mathrm{~h}$ at room temperature. Immunoreactive proteins were incubated with horseradish peroxidase-conjugated mouse HRP-linked IgG antibody (1:700 dilution in TBSTT with 2\% dry milk) (GE Healthcare Biosciences) for $1 \mathrm{~h}$ at room temperature. After washing with TBST, the reactants were developed using the enhanced chemiluminescence kit (GE Healthcare Biosciences).

Co-immunoprecipitation and immunoblot analyses. The Co-immunoprecipitation analysis was performed as previously described (15). In briefly, H322 or H1650 cell lysate were immunoprecipitated overnight with $3 \mu \mathrm{g}$ of the Polyclonal Goat anti-Src antibody (AF3389, R\&D). Immunoprecipitates were collected on protein A/G beads (30 $\mu \mathrm{l}$ per immunoprecipitation). Beads were washed three times in lysis buffer, resuspended in $35 \mu \mathrm{l}$ of Laemmli sample buffer, and resolved on $7.5 \%$ SDS-polyacrylamide gels. Proteins were transferred 


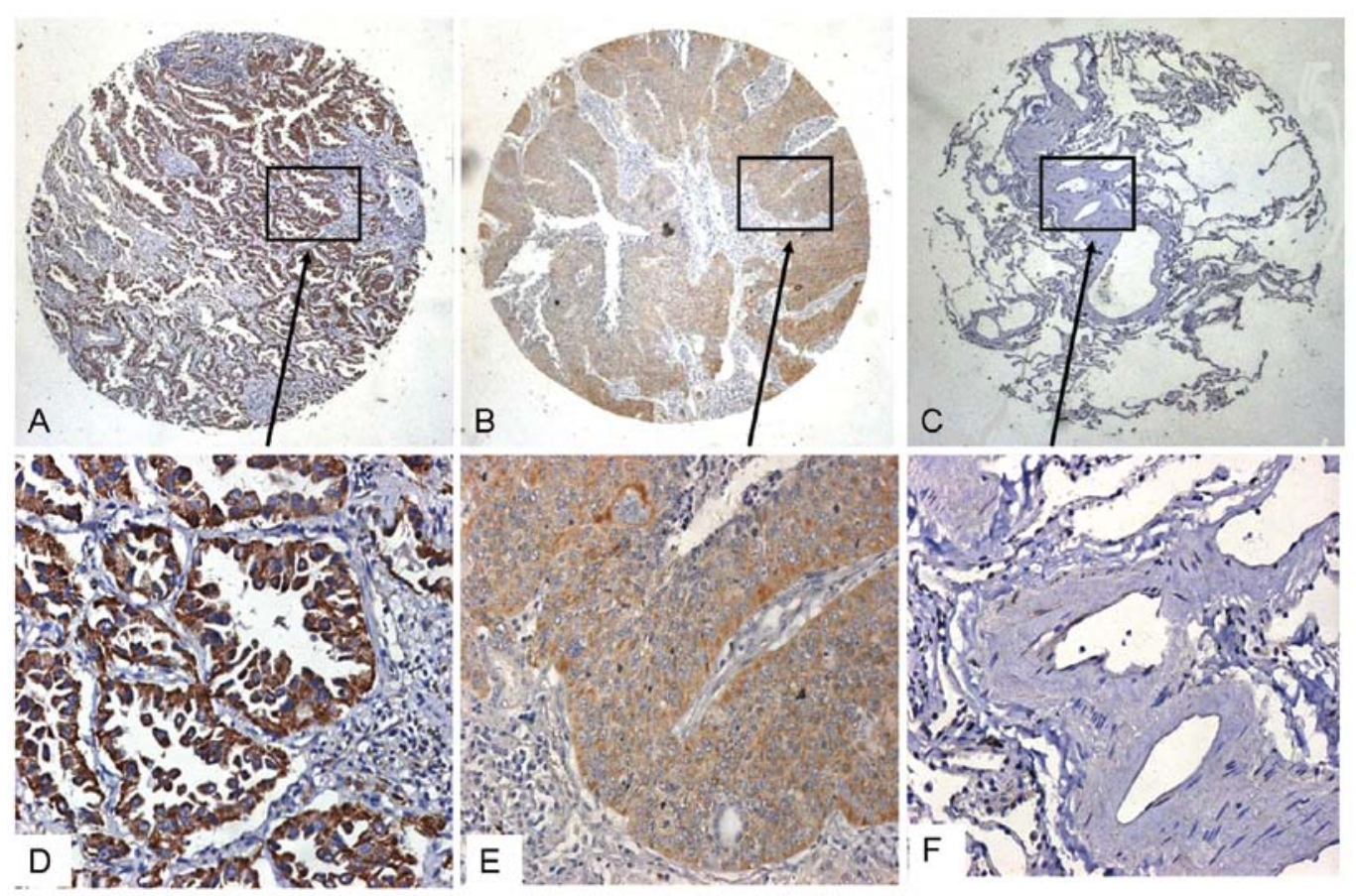

Figure 1. ARHGEF5 expression in tumor and adjacent tissues. The ARHGEF5 expression was determined by immunostaining. Expression of ARHGEF5 in ADC tissue (A, x50; D, x200), SCC tissue (B, x50; E, x200) and adjacent tissue (C, x50; F, x200).

to nitrocellulose membranes (Protran; Schleicher and Schuell), immunoblotted with the Polyclonal Rabbit anti-ARHGEF5 (11379-1-AP, Proteintech Group), and subjected to chemiluminescent detection.

Statistical analysis. SPSS 18.0 (SPSS Inc., Chicago, IL, USA) was used to perform the statistical analysis. Chi-square test and likelihood ratio test were used to illustrate the significance of various clinical characteristics. The $\chi^{2}$ test was used to assess the association between clinical features and the expression of biological factors. OS and the $95 \%$ confidence intervals (CIs) were evaluated by the Kaplan-Meier method comparing the different groups by log-rank test. The Cox proportional hazards model was used to evaluate the prognostic role of each single studied parameter on OS, in univariate and multivariate analyses. P-values were considered statistically significant at $<0.05$.

\section{Results}

Clinical characteristics of the patients. The patient clinical characteristics are summarized according to the histologic types (Table I). A total of 193 NSCLC patients (male, 145; female, 48; average age, 61.84 years; age range, 31-84) were enrolled in this study, of which 99 cases were squamous cell carcinoma (SCC) $(51.30 \%)$ and 94 cases were adenocarcinomas (ADC) (48.70\%). Of the patients enrolled in the study, 83 cases $(43.01 \%)$ had tumor metastasis in the lymph nodes and 110 cases $(56.99 \%)$ did not (Table I).

Expression of ARHGEF5 in NSCLC. As shown in Fig. 1, ARHGEF5 expression was detected in the ADC and SCC tissues, but not in the adjacent tissues. The ARHGEF5 was mainly located in the cytoplasma of tumor cells. In all 193 cases,
Table I. Characteristics of the NSCLC patients.

\begin{tabular}{lc}
\hline Characteristics & Cases $(\%)$ \\
\hline Histology & \\
SCC & $99(51.30)$ \\
ADC & $94(48.70)$ \\
Age (years) & \\
$<60$ & $70(36.27)$ \\
$\geq 60$ & $123(63.73)$ \\
Gender & \\
Male & $145(75.13)$ \\
Female & $48(24.87)$ \\
Pathologic Stage & \\
I & $68(35.23)$ \\
II & $61(31.61)$ \\
III & $64(33.16)$ \\
Tumor Grade & \\
I & $15(7.78)$ \\
II & $108(55.96)$ \\
III & $70(36.27)$ \\
Lymph node metastasis & \\
Positive & \\
Negative & \\
\hline
\end{tabular}

ARHGEF5 was positive in 133 cases $(68.91 \%)$ and negative in 60 cases $(31.09 \%$ ) (Table II). According to the histological subtypes, ARHGEF5 was detected in 64 of 94 cases of ADC 
Table II. Relationship between the clinical characteristics and the expression of ARHGEF5.

\begin{tabular}{|c|c|c|c|c|}
\hline Characteristics & $\begin{array}{c}\text { ARHGEF5 positive } \\
\text { cases }(\%)\end{array}$ & $\begin{array}{c}\text { ARHGEF5 negative } \\
\text { cases }(\%)\end{array}$ & Total & P-value \\
\hline \multicolumn{5}{|l|}{ Histology } \\
\hline $\mathrm{SCC}$ & $69(69.70)$ & 30 & 99 & \multirow[t]{2}{*}{0.931} \\
\hline $\mathrm{ADC}$ & $64(68.09)$ & 30 & 94 & \\
\hline \multicolumn{5}{|l|}{ Gender } \\
\hline Male & $103(71.03)$ & 42 & 145 & \multirow[t]{2}{*}{0.299} \\
\hline Female & $30(62.5)$ & 18 & 48 & \\
\hline \multicolumn{5}{|l|}{ Age (years) } \\
\hline$\geq 60$ & $78(63.41)$ & 45 & 123 & \multirow[t]{2}{*}{0.043} \\
\hline$<60$ & $55(78.57)$ & 15 & 70 & \\
\hline \multicolumn{5}{|l|}{ Differentiation } \\
\hline Well/ moderately & $71(57.72)$ & 52 & 123 & \multirow[t]{2}{*}{0.032} \\
\hline Poorly & $52(74.29)$ & 18 & 70 & \\
\hline \multicolumn{5}{|l|}{ Tumor stage } \\
\hline I & $39(57.35)$ & 29 & 68 & \multirow[t]{3}{*}{0.027} \\
\hline II & 48 (78.69) & 13 & 61 & \\
\hline III & $45(70.31)$ & 19 & 64 & \\
\hline \multicolumn{5}{|c|}{ Lymph node metastasis } \\
\hline Yes & $60(72.29)$ & 23 & 83 & \multirow[t]{2}{*}{0.469} \\
\hline No & $73(66.36)$ & 37 & 110 & \\
\hline
\end{tabular}

Table III. Correlation between ARHGEF5 and Src expression in the tumor tissues of NSCLC patients.

\begin{tabular}{llccrr}
\hline Subtypes & Group & ARHGEF5 (+) & ARHGEF5 (-) & Total & P-value \\
\hline NSCLC & Src (+) & 110 & 35 & 145 & 0.001 \\
& Src (-) & 23 & 25 & 48 & \\
& Total & 133 & 60 & 193 & 0.107 \\
SCC & Src (+) & 50 & 17 & 67 & 0.000 \\
& Src (-) & 18 & 14 & 99 & 77 \\
ADC & Total & 68 & 31 & 17 & \\
& Src (+) & 59 & 12 & 94 & \\
& Src (-) & 5 & 30 & & \\
& Total & 64 & & & \\
\end{tabular}

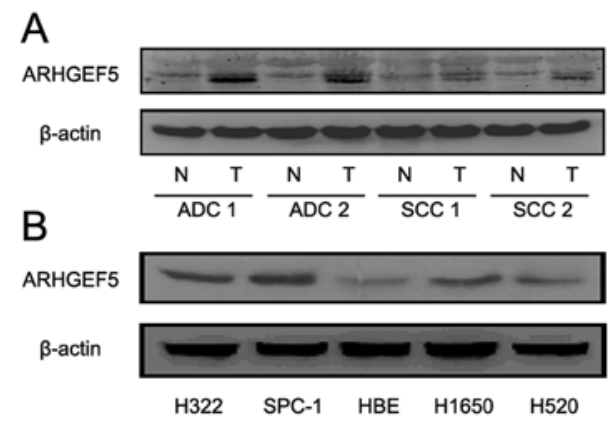

Figure 2. Western blot analysis of ARHGEF5 expression in the NSCLC tissues and lung cancer cell lines. (A) The ARHGEF5 expression in the NSCLC tissues (T) and adjacent normal tissues (N). (B) The ARHGEF5 expression in the lung cancer cell lines.
$(68.09 \%)$ and 69 of 99 cases of SCC (69.70\%) (Table II). The level of ARHGEF5 in poorly differentiated tumors was significantly higher than that in moderately-to-well differentiated tumors $(\mathrm{P}=0.032$, Table II). Younger patients $(<60$ years) also exhibited higher ARHGEF5 expression than older ones $(\mathrm{P}=0.043$, Table II). Furthermore, the level of ARHGEF5 was significantly associated with tumor stage $(\mathrm{P}=0.027$, Table II).

In addition, we examined the expression of ARHGEF5 in lung cancer tissues and NSCLC cell lines by western blotting. The level of ARHGEF5 protein was obviously higher in tumor tissues than in adjacent tissues (Fig. 2A). Similar results were observed in NSCLC cell lines than in human bronchoepithelial (HBE) cells (Fig. 2B). 


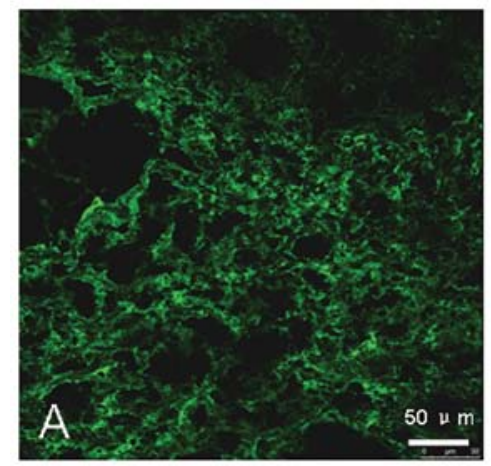

Src

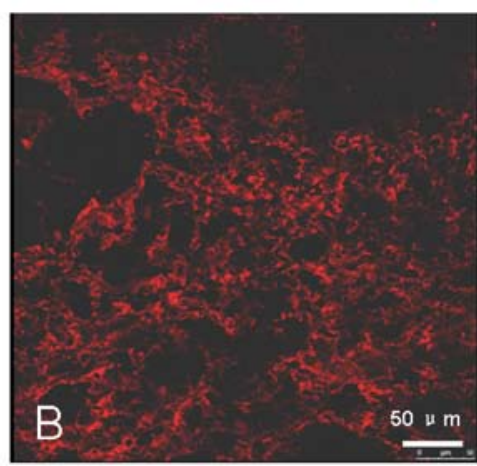

ARHGEF5

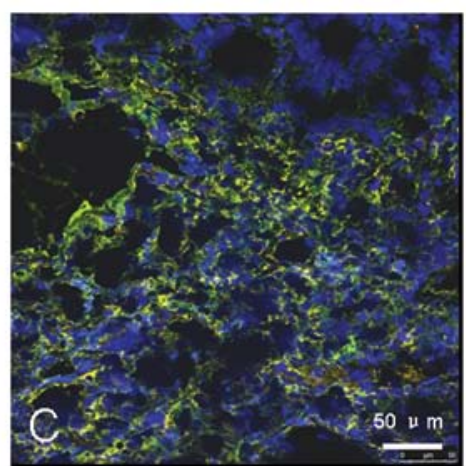

Merge

Figure 3. Lung cancer tissues were stained with anti-ARHGEF5 and anti-Src antibodies. Immunofluorescent staining of NSCLC tissues was performed with (A) anti-Src antibody or (B) anti-ARHGEF5 antibody. (C) Merged image. Scale bar $50 \mu \mathrm{m}$.

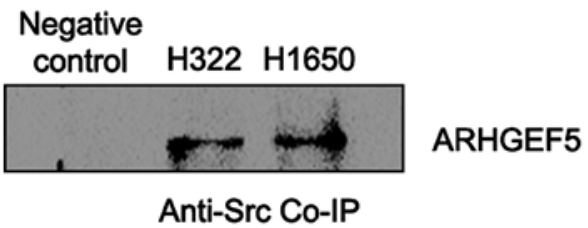

Figure 4. Western blot analysis of ARHGEF5 protein by anti-Src Co-IP.

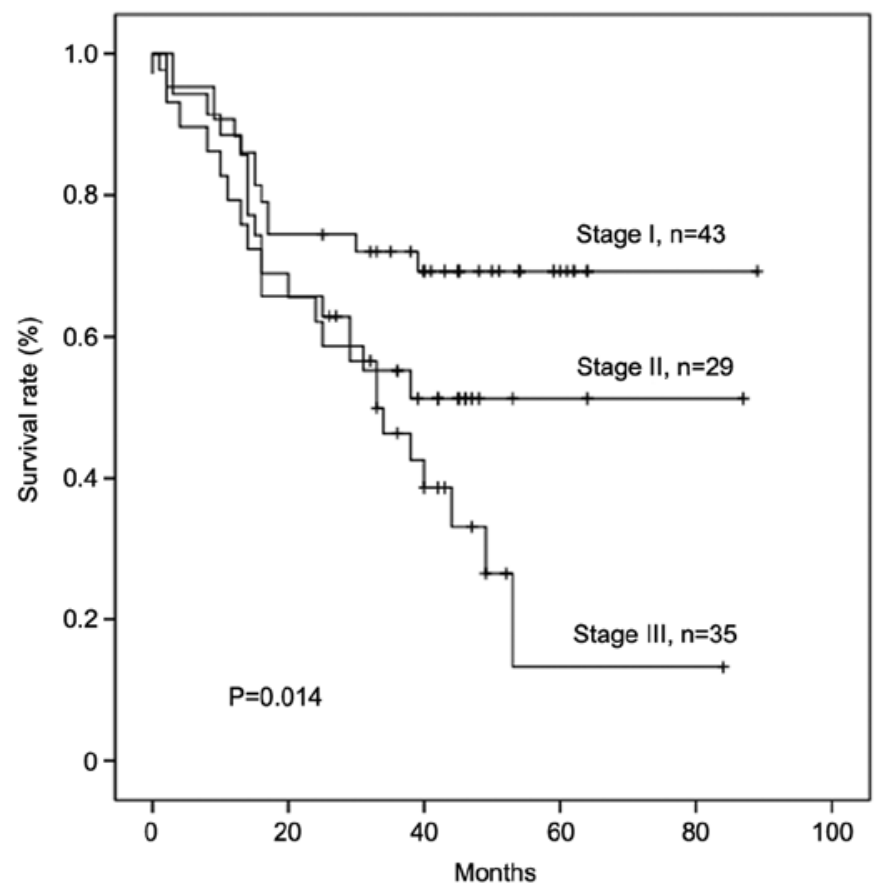

Figure 5. Kaplan-Meier survival plot by stage of 107 patients with resected NSCLC.

Expression of Src in NSCLC. As shown in Table III, among the 133 NSCLC patients with positive ARHGEF5 expression, 110 cases $(56.99 \%)$ showed positive Src expression, whereas 23 cases showed negative Src expression. In contrast, among the 60 patients with negative ARHGEF5, 25 patients showed negative Src expression, whereas 35 patients showed positive Src protein expression. Among the 64 ADC patients with positive ARHGEF5 expression, 59 showed positive Src expression, whereas 5 patients had negative Src expression. In contrast, among the 30 patients with negative ARHGEF5, 12 patients showed negative Src expression, whereas 18 patients had positive Src protein expression. A significant correlation between ARHGEF5 and Src expression in NSCLC $\left(\chi^{2}=11.874, \mathrm{P}=0.001\right)$ and in $\operatorname{ADC}\left(\chi^{2}=12.194, \mathrm{P}=0.000\right)$ was found. However, there was no correlation between ARHGEF5 and Src expression in SCC.

ARHGEF5 interacts with Src directly in NSCLC. To confirm the interaction between ARHGEF5 and Src, fluorescent immunohistochemistry and co-immunoprecipitation were performed with the tumor tissues and lung cancer cell lines, respectively. It was shown that ARHGEF5 and Src were co-expressed in NSCLC patient tumor tissues (Fig. 3) and the interaction between Src and ARHGEF5 was revealed by co-immunoprecipitation in NCI-H322 cells and NCI-H1650 cells (Fig. 4).

Survival analysis. A total of 107 patients completed the overall survival (OS) follow-up and 86 patients were lost to follow-up. The median follow-up time was 34.29 months. There was a significant difference in the OS time of patients in different clinical stages (Fig. 5). To further explore whether there was a correlation between the co-expression of ARHGEF5 and Src and OS time, the patients were stratified into 2 groups according to ARHGEF5 and Src expression: group 1, ARHGEF5(+)/Src(+) patients $(n=56)$; group 2, other patients $(\mathrm{n}=51)$. The median survival times for group 1 and group 2 were 29.37 months and 39.90 months, respectively. The patients in ARHGEF5(+)/Src(+) group showed a worse OS compared with the other patients (29.37 months versus 39.90 months, $\mathrm{P}=0.029$, Fig. 6).

\section{Discussion}

In the present study, we assessed the expression of ARHGEF5 in NSCLC tissues and the possible correlation of ARHGEF5 and Src co-expression with the survival time of NSCLC patients. The results revealed a significant increase of ARHGEF5 expression in NSCLC tissues and cultured cell lines compared with in matched adjacent tissues and HBE 


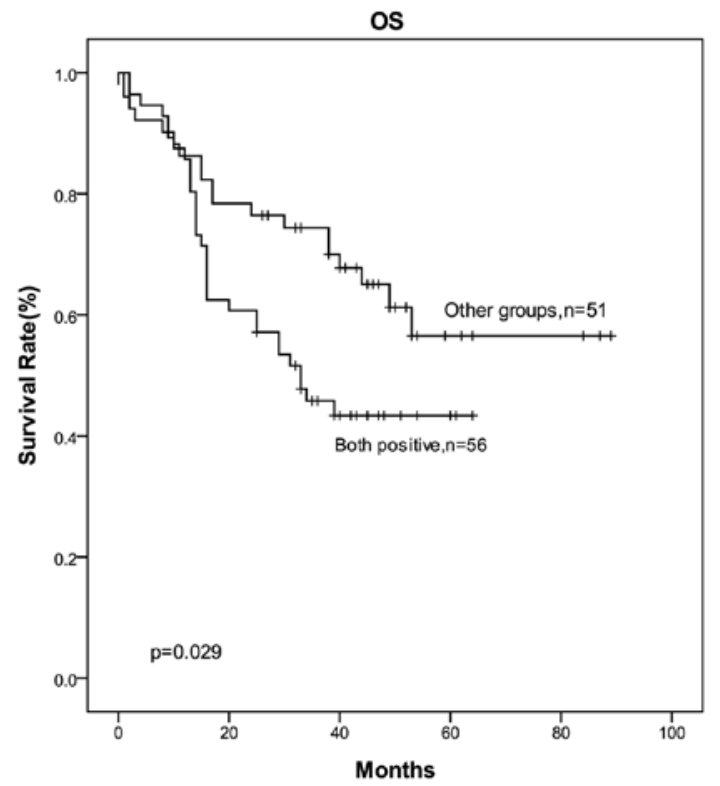

Figure 6. The overall survival rate between the both ARHEGF5/Src positive, and the other patients.

cells, respectively. Notably, an interaction between ARHGEF5 and Src was detected in both NSCLC tissues and cultured lung cancer cells. Among the cases completing the OS follow-up, the patients with ARHGEF5(+)/Src(+) showed a worse OS time compared with the other patients.

In the development and progression of human lung cancer, many recent studies have revealed a link with the deregulation of GEFs (16). The expression of Rac1-GEF Tiam1 is higher in high-metastatic cells than in low-metastatic cells, and downregulation of Tiam 1 can reduce the invasiveness of highmetastatic human giant-cell lung cancer cells (17). ECT2, a member of the human Dbl family GEFs, is overexpressed in the lung and esophageal cancers. Furthermore, high level of ECT2 was found to be associated with poor prognosis of lung cancer patients (18). Aberrant expression of Vav1 was found in $42 \%$ of 78 lung cancer cell lines examined and $46 \%$ of 57 human primary lung cancer specimens and the depletion of Vav1 expression in H358 and H441 lung cancer cells resulted in the reduction of colony formation in soft agar in vitro and tumor size in nude mice $(19,20)$. A recent study showed that Vav2- and Vav3-deficiency reduced microvascular density, tumor cell growth and survival in the transplanted B16 melanoma and Lewis lung carcinoma models in vivo (21). In the present study, we showed that ARHGEF5 was also highly expressed in the resected NSCLC tissues and cultured lung cancer cell lines. In addition, we showed that ARHGEF5 was closely correlated with age, tumor stage and differentiation. It is well known that younger NSCLC patients usually display a more aggressive disease course $(22,23)$. In the present study, we found that the expression of ARHGEF5 in young patients ( $<60$ years) was significantly higher than that in older patients ( $>60$ years). Since the tumor stage and differentiation had a high correlation with the prognosis, the level of ARHGEF5 might correlate with the prognosis of NSCLC patients. Thus, the overexpression of ARHGEF5 in young patients may be related to the aggressive disease course.
High levels of Src kinase activity, which can promote tumor cell growth and differentiation $(24,25)$ have been observed in many cancer types, including lung cancer (26-28). Src may stimulate tumorigenesis in NSCLC in a variety of ways, such as the signal transducer and activator of transcription 3 (STAT-3) and focal adhesion kinase (FAK)-related pathways, both of which are involved in tumor survival $(29,30)$. Src activates the VEGF pathway via STAT-3 (31) and in response to hypoxia in human lung adenocarcinoma cells, thus increasing the blood supply to the oxygen-starved tumor (32). In this study, we found that Src was extensively expressed in NSCLC tissues. Then we further analyzed the co-expression of ARHGEF5 and Src in SCC and ADC, the results showed that the positive rate of ARHGEF5 and Src co-expression was higher in ADC (62.77\%) than in SCC $(50.51 \%)$ and the expression of ARHGEF5 was associated with the expression of $\operatorname{Src} \operatorname{ADC}\left(\chi^{2}=12.194, \mathrm{P}=0.000\right)$, but not in SCC. Furthermore, it has been reported that ARHGEF5 is able to be phosphorylated by Src and then bound to Src to form a complex with Src and PI3K (12). Our results showed that there was a dynamic interaction between ARHGEF5 and Src in the lung cancer tissues and lung cancer cell lines, indicating that the Src-ARHGEF5 pathway may play a role in the tumorigenesis and development of ADC rather than SCC.

To explore the clinical significance of co-expression of ARHGEF5 and Src in patients with resected NSCLC, we analyzed the correlation of ARHGEF5 and Src co-expression and the OS in NSCLC patients. Noteworthy, we found patients with ARHGEF5(+)/Src(+) had a significantly shorter OS than the other patients. Therefore, ARHGEF5/ Src can be considered a candidate prognostic biomarker for patients with resected NSCLC. Furthermore, activation of STAT-3 and FAK by Src is required for anchoragedependent and -independent cell growth in a range of human tumors including NSCLC (30). Therefore, Src, a cellular tyrosine kinase, offers a particularly promising molecular target for anticancer therapy, as inhibition of Src may lead to the inhibition of multiple signaling pathways, and thereby the inhibition of tumor progress. However, although a number of Src inhibitors are currently being investigated as potential therapies for NSCLC, such as dasatinib $(11,33)$, the antitumor activity of Src inhibitors is limited. Thus, the combination with other signal protein inhibitors is often needed (34). In the present study, we showed that ARHGEF5 was overexpressed in the lung cancer tissues and correlated with tumor stage and differentiation. In addition, co-expression of ARHGEF5 and Src was correlated with the prognosis of NSCLC patients and there was an interaction between ARHGEF5 and Src in the lung cancer tissues. These findings suggest that inhibition of both Src and ARHGEF5 in NSCLC may be a promising cancer therapy for patients with resected NSCLC.

In conclusion, our study found an obvious expression of ARHGEF5 in the NSCLC tissues and lung cancer cell lines. The expression of ARHGEF5 correlated with age, tumor stage and the differentiation. In addition, Src was also expressed in the NSCLC tissues, and co-expression of Src and ARHGEF5 correlated with shorter OS time, indicating ARHGEF5/Src can be considered as a prognostic biomarker and a therapeutic target for patients with resected NSCLC. 


\section{Acknowledgements}

This study was supported by the National Natural Science Foundation of China (No. 81001045).

\section{References}

1. Goya T, Asamura H, Yoshimura H, et al: Prognosis of 6644 resected non-small cell lung cancers in Japan: a Japanese lung cancer registry study. Lung Cancer 50: 227-234, 2005.

2. Lee SY, Kim MJ, Jin G, et al: Somatic mutations in epidermal growth factor receptor signaling pathway genes in non-small cell lung cancers. J Thorac Oncol 5: 1734-1740, 2010.

3. Casas-Selves M, Kim J, Zhang Z, et al: Tankyrase and the canonical Wnt pathway protect lung cancer cells from EGFR inhibition. Cancer Res 72: 4154-4164, 2012.

4. Ye YZ, Zhang ZH, Fan XY, et al: Notch3 overexpression associates with poor prognosis in human non-small-cell lung cancer. Med Oncol 30: 595, 2013

5. Chan AM, McGovern ES, Catalano G, Fleming TP and Miki T: Expression cDNA cloning of a novel oncogene with sequence similarity to regulators of small GTP-binding proteins. Oncogene 9: 1057-1063, 1994

6. Takai S, Chan AM, Yamada K and Miki T: Assignment of the human TIM proto-oncogene to 7q33-->q35. Cancer Genet Cytogenet 83: 87-89, 1995.

7. Zheng Y: Dbl family guanine nucleotide exchange factors Trends Biochem Sci 26: 724-732, 2001.

8. Hart MJ, Eva A, Evans T, Aaronson SA and Cerione RA: Catalysis of guanine nucleotide exchange on the CDC42Hs protein by the dbl oncogene product. Nature 354: 311-314, 1991.

9. Debily MA,Camarca A, Ciullo M, et al: Expression and molecular characterization of alternative transcripts of the ARHGEF5/TIM oncogene specific for human breast cancer. Hum Mol Genet 13 323-334, 2004.

10. Xie X, Chang SW, Tatsumoto T, Chan AM and Miki T: TIM a Dbl-related protein, regulates cell shape and cytoskeletal organization in a Rho-dependent manner. Cell Signal 17: 461-471, 2005.

11. Giaccone G and Zucali PA: Src as a potential therapeutic target in non-small-cell lung cancer. Ann Oncol 19: 1219. 1223,2008

12. Kuroiwa M, Oneyama C, Nada S and Okada M: The guanine nucleotide exchange factor Arhgef5 plays crucial roles in Src-induced podosome formation. J Cell Sci 124: 1726-1738, 2011.

13. Li P, Zhang Z, Wang Q, et al: The ectopic expression of IFN regulatory factor 4-binding protein is correlated with the malignant behavior of human breast cancer cells. Int Immunopharmacol 9: 1002-1009, 2009.

14. Maaser K, Daubler P, Barthel B, et al: Oesophageal squamous cell neoplasia in head and neck cancer patients: upregulation of COX-2 during carcinogenesis. Br J Cancer 88: 1217-1222, 2003.

15. Sharma SV, Oneyama C, Yamashita Y, et al: UCS15A, a nonkinase inhibitor of Src signal transduction. Oncogene 20 2068-2079, 2001.

16. Lazer $\mathrm{G}$ and Katzav $\mathrm{S}$ : Guanine nucleotide exchange factors for RhoGTPases: good therapeutic targets for cancer therapy? Cell Signal 23: 969-979, 2011.
17. Hou M, Tan L, Wang X and Zhu YS: Antisense Tiam1 downregulates the invasiveness of $95 \mathrm{D}$ cells in vitro. Acta Biochim Biophys Sin 36: 537-540, 2004.

18. Hirata D, Yamabuki T, Miki D, et al: Involvement of epithelial cell transforming sequence-2 oncoantigen in lung and esophageal cancer progression. Clin Cancer Res 15: 256-266, 2009.

19. Fernandez-Zapico ME, Gonzalez-Paz NC, Weiss E, et al: Ectopic expression of VAV1 reveals an unexpected role in pancreatic cancer tumorigenesis. Cancer Cell 7: 39-49, 2005.

20. Lazer G, Idelchuk Y, Schapira V, Pikarsky E and Katzav S: The haematopoietic specific signal transducer Vav1 is aberrantly expressed in lung cancer and plays a role in tumourigenesis. J Pathol 219: 25-34, 2009.

21. Brantley-Sieders DM, Zhuang G, Vaught D, et al: Host deficiency in Vav2/3 guanine nucleotide exchange factors impairs tumor growth, survival, and angiogenesis in vivo. Mol Cancer Res 7: 615-623, 2009.

22. Serrano-Olvera A and Gerson R: Age associated survival rate in non small cell lung cancer. Gac Med Mex 145: 27-35, 2009 (In Spanish).

23. Salmeron D, Chirlaque MD, Isabel Izarzugaza M, et al: Lung cancer prognosis in Spain: the role of histology, age and sex. Respir Med 106: 1301-1308, 2012.

24. Xu W, Allbritton $\mathrm{N}$ and Lawrence DS: SRC kinase regulation in progressively invasive cancer. PLoS One 7: e48867, 2012.

25. Budde RJ, Ke S and Levin VA: Activity of pp60c-src in 60 different cell lines derived from human tumors. Cancer Biochem Biophys 14: 171-175, 1994.

26. Mazurenko NN, Kogan EA, Zborovskaya IB and Kisseljov FL: Expression of pp60c-src in human small cell and non-small cell lung carcinomas. Eur J Cancer 28: 372-377, 1992.

27. Masaki T, Igarashi $\mathrm{K}$, Tokuda $\mathrm{M}$, et al: pp60c-src activation in lung adenocarcinoma. Eur J Cancer 39: 1447-1455, 2003.

28. Thomas SM and Brugge JS: Cellular functions regulated by Src family kinases. Annu Rev Cell Dev Biol 13: 513-609, 1997.

29. Laird AD, Li G, Moss KG, et al: Src family kinase activity is required for signal tranducer and activator of transcription 3 and focal adhesion kinase phosphorylation and vascular endothelial growth factor signaling in vivo and for anchorage-dependent and -independent growth of human tumor cells. Mol Cancer Ther 2: 461-469, 2003

30. Song L, Turkson J, Karras JG, Jove R and Haura EB: Activation of Stat 3 by receptor tyrosine kinases and cytokines regulates survival in human non-small cell carcinoma cells. Oncogene 22: 4150-4165, 2003.

31. Eliceiri BP, Paul R, Schwartzberg PL, Hood JD, Leng J and Cheresh DA: Selective requirement for Src kinases during VEGF-induced angiogenesis and vascular permeability. Mol Cell 4: 915-924, 1999

32. Sato M, Tanaka T, Maeno T, et al: Inducible expression of endothelial PAS domain protein-1 by hypoxia in human lung adenocarcinoma A549 cells. Role of Src family kinasesdependent pathway. Am J Respir Cell Mol Biol 26: 127-134, 2002.

33. Johnson FM, Bekele BN, Feng L, et al: Phase II study of dasatinib in patients with advanced non-small-cell lung cancer. J Clin Oncol 28: 4609-4615, 2010

34. Haura EB, Tanvetyanon T, Chiappori A, et al: Phase I/II study of the Src inhibitor dasatinib in combination with erlotinib in advanced non-small-cell lung cancer. J Clin Oncol 28: 1387-1394, 2010. 